\title{
ANÁLISIS DE SENDERO EN BERENJENA (Solanum melongena L.)
}

\section{PATH ANALYSIS IN EGGPLANT (Solanum melongena L.)}

\author{
Hermes Aramendiz-Tatis ${ }^{1}$, Miguel Espitia², Carlos Cardona ${ }^{3}$
}

${ }^{1}$ Profesor titular, Facultad de Ciencias Agrícolas, Universidad de Córdoba, Montería. haramendiz@sinu.unicordoba.edu. co - Ciudad Universitaria Carrera 6 No. 76-103. Código Postal: 354. Montería - Colombia; ${ }^{2}$ Profesor titular, Facultad de Ciencias Agrícolas, Universidad de Córdoba, Montería. mespitia@sinu.unicordoba.edu.co 3Profesor titular, Facultad de Ciencias Agrícolas, Universidad de Córdoba, Montería. ccardona@sinu.unicordoba.edu.co

Rev. U.D.C.A. Act. \& Div. Cient. 13 (1): 115-123, 2010

\section{RESUMEN}

La berenjena es de gran importancia en el caribe colombiano, por seguridad alimentaria, generación de ingresos y empleo, beneficios en salud y grandes posibilidades de exportación; sin embargo, la casi totalidad de los genotipos que se siembran son criollos y poco mejorados. En Colombia, no se han reportado estudios sobre análisis de sendero para maximizar el progreso, por selección en el mejoramiento de esta especie. Veinticuatro genotipos de berenjena de diferente origen geográfico fueron estudiados en el Centro de Investigación Turipaná de Corpoica (Cereté - Colombia), utilizando el diseño de bloques completamente al azar con tres repeticiones, con el objetivo de determinar los efectos directos e indirectos de los componentes del rendimiento sobre la producción de frutos. El análisis de sendero reveló, que en primera instancia, el número de frutos por plantas, seguido del peso de frutos, tienen un efecto directo sobre el rendimiento de frutos. Por lo tanto, el mejoramiento genético simultáneo de berenjena debe considerar estos dos caracteres como un límite fisiológico.

Palabras clave: Mejoramiento, correlaciones, selección individual, rendimiento.

\section{SUMMARY}

Eggplants are of great importance in the Colombian Caribbean Coast for food sucurity, income and employment, health benefits and large export potential, but most of the genotypes planted are landraces and poorly bred. In Colombia no reportes about path analysis to improve selection for eggplant breeding exist. Twentyfour eggplant genotypes from different geographical origin were studied in the Corpoicas Research Center Turipaná (Cereté - Colombia), using a complete randomized block design with three replicates, with the objetive to determine the direct and indirect effects of yield components on the fruit production. The path analysis revealed that the number of fruits per plant, followed by fruit weight have a direct signifficant effect on fruit yield. Therefore, the simultaneous breeding of eggplant must considers these two characters as a physiological limit.

Key words: Breeding, correlations, individual selection, yield.

\section{INTRODUCCIÓN}

La berenjena es una de las hortalizas de gran importancia en el caribe colombiano y con grandes posibilidades de exportación, por sus beneficios en la salud, por su alto contenido de fenoles, con actividad antioxidante y minerales, como P, K, Ca y Mg; su aporte a la dieta en calorías es reducido, tiene muy bajo contenido en sodio y representa una buena fuente de fibra (Raigon et al. 2008; Palomo et al.2009).

En los departamentos de Córdoba, Sucre y Bolívar, se cultivan 374 ha que representan el $72 \%$ de la producción 
nacional. El rendimiento de los cultivares regionales alcanza las $16 \mathrm{t} . \mathrm{ha}^{-1}$ y se siembra en superficies que oscilan entre 1000 y $2500 \mathrm{~m}^{2}$, por productores minifundistas, campesinos sin tierras y jornaleros en actividades agropecuarias (Araméndiz et al. 1999; DNP, 2005; Agronet, 2008). En tanto, que el rendimiento de los híbridos frecuentemente supera las $50 \mathrm{t}^{-h a^{-1}}$ y se caracterizan por ser precoces, uniformes a la cosecha y de mejores características para el almacenamiento (Sekara et al. 2007).

Desde la incorporación de la berenjena a la actividad agrícola del departamento de Córdoba, pocos son los esfuerzos en desarrollar cultivares de alto rendimiento, calidad de fruto y adaptabilidad a las áreas productoras de la costa atlántica, razón por la cual aún se siguen produciendo los cultivares tradicionales, como criolla lila, criolla blanca y criolla morada; sin embargo, estas poblaciones poseen características de interés, como adaptación, color de fruto, altura de planta, tolerancia a factores bióticos, tamaño de fruto, resistencia a estrés por sequía, que son importantes en un programa de mejoramiento.

Los métodos modernos de mejoramiento genético existentes hoy en día permiten obtener variedades e híbridos de alto rendimiento, que posean otros atributos agronómicos, favorables al productor y al consumidor (Hatipoğlu, 1993; Martínez E Torregroza, 1988). El rendimiento es una característica poligénica compleja que depende directa o indirectamente de otras características conocidas como componentes del rendimiento.

Los coeficientes de correlación representan un indicativo de la asociación entre el rendimiento y sus componentes; su estimación ayuda al mejorador a entender su relación con estos, pero no destaca con claridad la contribución directa e indirecta de cada una de las características con el rendimiento (Bhatt, 1973). En este sentido, es interesante que el fitomejorador conozca las relaciones entre el rendimiento y los caracteres necesarios en la producción de un nuevo genotipo, siguiendo un método de selección en el que intervengan, simultáneamente, dos o más factores de importancia agronómica (Martínez E Torregroza, 1988). Por tal razón, es de mucha relevancia conocer la importancia relativa de cada uno de las características que afectan el rendimiento, a través del análisis de sendero.
El análisis de sendero es importante, porque permite descomponer las correlaciones de las variables independientes respecto a variables dependientes, como el rendimiento en sus respectivos efectos directos e indirectos. Así mismo, es de ayuda para el fitomejorador en la identificación de caracteres que pueden ser usados como criterio de selección, en un programa de mejoramiento de berenjena (Espitia et al. 2008a; Habib et al. 2007). Este tipo de análisis ha sido aplicado en ají (Legesse et al. 1999; Tavares et al. 1999; De Carvalho et al.1999); berenjena (Ingale \& Patil, 1995) y en melón (Krishna et al. 2007).

El estudio tuvo como propósito generar información básica sobre efectos directos e indirectos entre los componentes primarios del rendimiento y el rendimiento total de frutos de berenjena, para su aplicación en el programa de mejoramiento genético de esta especie.

\section{MATERIALES Y MÉTODOS}

Veinticuatro genotipos de berenjena originarias de Brasil, Colombia, Japón, Taiwán y Estados Unidos fueron cultivadas en Corpoica, en el C.I. Turipaná de Cereté (Córdoba - Colombia) durante el primer semestre de 2008 (Tabla 1). Sus coordenadas geográficas corresponden a los $8^{\circ} 31^{\prime} 16^{\prime \prime}$ de latitud norte y $75^{\circ} 58^{\prime} 11^{\prime}$ de longitud oeste. El área pertenece a la zona climática cálidomoderada, a la formación Bosque Seco Tropical (BS-T), zona agroecológica $\mathrm{C}_{\mathrm{j}}$; el suelo del área experimental es de textura franco arcillosa, con densidad aparente de $1,12 \mathrm{~g}$ $\mathrm{cm}^{3}$ y estructura moderadamente estable, clasificado taxonómicamente como Fluvaquentic endoaquept franco con promedios de 2,2\% de materia orgánica y $\mathrm{pH}$ de 5,9 (Palencia et al. 2006).

Los genotipos fueron evaluados utilizando el diseño experimental de bloques completamente al azar con tres repeticiones (bloques). El factor de bloqueo fue la pendiente. Cada unidad experimental fue constituida por un surco de $10 \mathrm{~m}$ de longitud, con distancia entre plantas y surcos de $1 \mathrm{~m}$, para una población de once plantas. El manejo agronómico, a nivel de semillero y campo, se realizó aplicando las recomendaciones dadas por Aramendiz et al. (2008), para el cultivo de la berenjena en el valle del Sinú.

Las variables de respuesta consideradas en el estudio corresponden a longitud del fruto (Lonfru), número de frutos por planta (Nufrupta), peso de fruto (Pefru), 
resistencia del fruto a la penetración (Resfru), altura de planta (Alpta) y rendimiento de frutos (Renfru) y fueron medidas en tres plantas por repetición, tomadas al azar, en cada una de las variedades. El rendimiento, se obtuvo sobre todas las plantas del surco.

El análisis de varianza y de covarianza para los seis caracteres, al igual que las correlaciones fenotípicas, ambientales y genotípicas y análisis de sendero, se llevaron a cabo mediante el uso de programa computacional Genes versión Window (2004.2.1), desarrollado por Cruz (2004).
El programa aplica las fórmulas clásicas de correlación:

1. Correlación fenotípica $(\mathrm{rF}(\mathrm{xy})) ; \mathrm{rF}(\mathrm{xy}))=\mathrm{COVF}(\mathrm{xy})$ / $\mathrm{SF}(\mathrm{x}) \cdot \mathrm{SF}(\mathrm{y})$

2. Correlación genética $(\mathrm{rG}(\mathrm{XY})) ; \mathrm{rG}(\mathrm{XY})=\mathrm{COVG}(\mathrm{XY})$ / SG(x). SG(y)

3. Correlación ambiental ( $\mathrm{rE}(\mathrm{XY}))$; $\mathrm{rE}(\mathrm{XY})=$ $\operatorname{COVE}(X Y) / \mathrm{SE}(\mathrm{x})$. SE (y)

En donde: $\mathrm{r}(\mathrm{XY})$ y COV (XY) son las correlaciones y covarianzas fenotípicas, genéticas y ambientales,

Tabla 1. Genotipos y procedencia de los cultivares de berenjena utilizados en el estudio.

\begin{tabular}{|c|c|c|}
\hline Código* & Genotipos & Procedencia \\
\hline BR01 & Broxa & TOPSEED-Brasil \\
\hline BR02 & Berenjena cica & EMBRAPA-Brasil \\
\hline BR03 & Linea tradicional & FELTRIN - Brasil \\
\hline $\mathrm{CC} 01$ & B. Long purple & FERCON - Colombia \\
\hline $\mathrm{CCO} 2$ & Black Bell & MIGUEL SAEN Y CIA. - Colombia \\
\hline $\mathrm{CCO3}$ & Berenjena Barcelona & IMPULSEMILLAS - Colombia \\
\hline $\mathrm{CCO} 4$ & Berenjena $N^{0} 5$ & IMPULSEMILLAS - Colombia \\
\hline $\mathrm{CC} 06$ & B. Long purple & ARROYAVE - Colombia \\
\hline $\mathrm{CC} 08$ & B. roxa & SEMICOL - Colombia \\
\hline JP01 & B. Japonesa SENRYO & JAPÓN \\
\hline TW01 & S. CHAOYAN TAIWAN & TAIWAN \\
\hline TW03 & BRINJAL MEBH-11 & TAIWAN \\
\hline TW04 & MANDHARI SEEDS & TAIWAN \\
\hline TW06 & SHUANGFEENG G.13 & TAIWAN \\
\hline EU01 & Black beauty & ESTADOS UNIDOS \\
\hline EU02 & Long purple & ESTADOS UNIDOS \\
\hline $\mathrm{C002}$ & Lila & CERETÉ, CÓRDOBA, COLOMBIA \\
\hline $\mathrm{CO03}$ & Lila pompa & CERETÉ, CÓRDOBA, COLOMBIA \\
\hline C009 & Morada & SAN CARLOS, CÓRDOBA, COLOMBIA \\
\hline $\mathrm{C} 016$ & Lila & CERETÉ, CÓRDOBA, COLOMBIA \\
\hline $\mathrm{CO23}$ & Berenjena Palanca & CERETÉ, CÓRDOBA, COLOMBIA \\
\hline $\mathrm{CO25}$ & Roja calabaza & CERETÉ, CÓRDOBA, COLOMBIA \\
\hline $\mathrm{C033}$ & Morada con espina & LORICA; CÓRDOBA, COLOMBIA \\
\hline $\mathrm{CO43}$ & Negra larga & MONTERÍA, CÓRDOBA, COLOMBIA \\
\hline
\end{tabular}


entre los caracteres X e Y, respectivamente; $\mathrm{S}(\mathrm{x})$ y $\mathrm{S}(\mathrm{y})$ son la desviaciones estándar fenotípicas, genéticas y ambientales de X e Y, respectivamente.

Una vez estimados los coeficientes de correlación, se confirmó la significancia estadística para cada " $r$ ", planteando la hipótesis nula: Ho: $r=0$, versus la hipótesis alterna: Ha: $r \neq 0$, mediante una prueba de $\mathrm{T}$, dada por la siguiente fórmula:

$$
T_{c}=\frac{r \sqrt{(n-2)}}{\sqrt{\left(1-r^{2}\right)}}
$$

La "T" calculada (Tc), se comparó con una T tabla (Tt), al nivel de significancia seleccionado 0.05 y 0.01 y con (n - 2) grados de libertad. La regla de decisión fue sí $\mathrm{Tc} \geq \mathrm{Tt}$, entonces el valor de " $\mathrm{r}$ " es estadísticamente diferente de cero.

Se realizaron dos análisis de sendero para el sistema: Renfru, como variable efecto $(\mathrm{Y})$, en función de las variables causas: Lonfru $\left(\mathrm{X}_{1}\right)$, Nufrupta $\left(\mathrm{X}_{2}\right)$, Pefru $\left(\mathrm{X}_{3}\right)$ y Alpta $\left(\mathrm{X}_{4}\right)$. Los dos análisis de sendero, se originan del uso de las correlaciones fenotípicas y genotípicas entre tales variables. Ambas matrices las genera, de manera automática, el programa GENES, en el análisis de varianza.
Para estimar los efectos directos en cada uno de los análisis de sendero, GENES utiliza una matriz de correlación (fenotípica o genética, dependiendo del interés), la descompone y la organiza en el siguiente sistema de matrices: $P=A^{-1}$. $R$; en donde: $A^{-1}$ es la inversa de la matriz de correlaciones (entre cada una de las variables causas), $\mathrm{R}$ es el vector de coeficientes de correlaciones entre las variables causas con la variable efecto y $\mathrm{P}$ es el vector coeficientes de sendero.

La descomposición de los coeficientes de correlación de cada una de las variables causa con la variable efecto $\left(r_{x_{i}}\right)$, en sus componentes efecto directo $\left(P_{i}\right)$ y el efecto indirecto $\left(E_{i}\right)$, permite, mediante el despeje de las siguientes ecuaciones, estimar los respectivos efectos indirectos de cada variable causa $\left(\mathrm{E}_{\mathrm{i}}\right)$ :

$r_{x 1 y}=P_{1}+E_{1}$ : para con Lonfru

$r_{x 2 y}=P_{2}+E_{2}$ : para con Nufrupta

$r_{x 3 y}=P_{3}+E_{3}$ : para con Pefru

$r_{x 4 y}=P_{4}+E_{4}$ : para con Alpta

El coeficiente de sendero, debido a los efectos residuales o a otras variables no consideradas en el estudio (h), la estima mediante la siguiente ecuación:

$$
h=\left[1-\left(P_{1 .} r_{x 1 y}\right)-\left(P_{2,} r_{x 2 y}\right)-\left(P_{3 .} r_{x 3 y}\right)-\left(P_{4 .} r_{x 4 y}\right)\right]^{1 / 2}
$$

Tabla 2. Análisis de varianza para seis caracteres de interés agronómico en berenjena (Solanum melongena L.).

\begin{tabular}{|c|c|c|c|c|c|c|c|}
\hline \multirow{2}{*}{ F de V } & \multirow{2}{*}{ GI } & \multicolumn{6}{|c|}{ Cuadrados medios } \\
\cline { 3 - 8 } & & $\begin{array}{c}\text { Lonfru } \\
(\mathrm{cm})\end{array}$ & $\begin{array}{c}\text { Nufrupta } \\
(\#)\end{array}$ & $\begin{array}{c}\text { Pefru } \\
(\mathrm{g})\end{array}$ & $\begin{array}{c}\text { Resfru } \\
(\text { newts })\end{array}$ & $\begin{array}{c}\text { Alpta } \\
(\mathrm{cm})\end{array}$ & $\begin{array}{c}\text { Renfru } \\
\left(\mathrm{kg} \cdot \mathrm{ha}^{-1}\right)\end{array}$ \\
\hline Bloques & 2 & 0,0904 & 0,023 & 567,79 & 25438,88 & 67,43 & 3,55 \\
\hline Genotipos & 23 & $38,51^{* *}$ & $6,76^{* *}$ & $25208,4^{* *}$ & $5165040,5^{* *}$ & $1388,48^{* *}$ & $84,55^{* *}$ \\
\hline Error & 46 & 1,27 & 0,08 & 428,15 & 52013,16 & 26,53 & 1,29 \\
\hline Total & 71 & 13,30 & 2,24 & 8459,49 & 3625,90 & 468,87 & 28,42 \\
\hline C.V.(\%) & & 6,49 & 10,47 & 9,25 & 6,29 & 6,30 & 9,48 \\
\hline
\end{tabular}

${ }^{* *}$ Altamente significativa $(P>0,01)$

Lonfru: Longitud de fruto; Nufrupta: Número de frutos por planta; Pefru: Peso de fruto; Resfru: Resistencia de fruto; Alpta: Altura de planta; Renfru: Rendimiento de fruto; 


\section{RESULTADOS Y DISCUSIÓN}

El análisis de varianza individual para todos los caracteres sometidos a estudio reveló diferencias altamente significativas $(p \leq 0,01)$, entre los genotipos estudiados (Tabla 2). Estos resultados indican que las diferencias obedecen a la condición genética intrínseca de cada cultivar. Los coeficientes de variación experimental por su bajo valor $(\leq 10,47 \%)$ revelan la existencia de una alta precisión experimental, lo que permite garantizar la validez de las conclusiones emitidas.

Las correlaciones fenotípicas, genéticas y ambientales están consignadas en la tabla 3, donde se aprecia que las correlaciones fenotípicas y genotípicas fueron semejantes y las ambientales muy reducidas, especialmente, en las de mayor magnitud, indicando que las correlaciones fenotípicas fueron poco afectadas por el ambiente y revelan efectos de asociación a nivel genético, como lo señalan Legesse et al. (1999); Silva \& Vieira (2008); Habib et al. (2007); Shivanna et al. (2007); Espitia et al. (2008a).
Los coeficientes de correlación fenotípica y genotípica entre el rendimiento de frutos y las otras características medidas, señalan que el Renfru acusó ausencia de correlación con Lonfru, Pefru, Resfru y Alpta, en tanto que mostró correlación positiva y altamente significativa respecto al Nufrupta $(0,56 * *)$. Estos resultados son coincidentes con los anotados por Kruiteva (1985); Vadivel \& Bapu (1988a, 1988b); Ingale \& Patil (1995); Yadad et al. (1997); Lohakare et al. (2008) y Bansal \& Metha (2008), excepto entre el rendimiento de fruto y peso de fruto que, en este estudio, acusó ausencia de asociación.

La ausencia de correlación significativa entre Renfru y Pefru, al igual que con Lonfru (Tabla 3), ha sido registrado como significativas y positivas por otros investigadores, como Mak E Vijiarungam (1980) en berenjena y con Pefru (De Carvalho et al. 1999), en pimentón. Ello, posiblemente obedece a la presencia de efectos indirectos enmascarados que tienen otras variables, sobre el nivel de asociación entre tales pares de caracteres, ya que al realizar el análisis de correlación

Tabla 3. Correlaciones fenotípicas $\left(r_{F}\right)$, genéticas $\left(r_{G}\right)$ y ambientales $\left(r_{E}\right)$ para seis caracteres de berenjena, en el valle del Sinú.

\begin{tabular}{|l|c|c|c|c|c|c|}
\hline & $r^{\prime} s$ & Nufrupta & Pefru & Renfru & Resfru & Alpta \\
\hline Lonfru & $r_{F}$ & $-0,33$ & 0,39 & 0,00 & $-0,67^{* *}$ & $0,52^{* *}$ \\
\hline & $r_{G}$ & $-0,35$ & 0,31 & 0,00 & $-0,68^{* *}$ & $0,53^{* *}$ \\
\hline & $r_{E}$ & 0,22 & $-0,07$ & 0,10 & 0,04 & 0,14 \\
\hline Nufrupta & $r_{F}$ & & $-0,63^{* *}$ & $0,56^{* *}$ & $-0,02$ & $-0,47^{*}$ \\
\hline & $r_{G}$ & & $-0,64^{* *}$ & $0,56^{* *}$ & $-0,02$ & $-0,46^{*}$ \\
\hline & $r_{E}$ & & 0,04 & 0,23 & $-0,04$ & $-0,02$ \\
\hline Pefru & $r_{F}$ & & & 0,04 & $-0,06$ & $0,74^{* *}$ \\
\hline & $r_{G}$ & & & 0,04 & $-0,06$ & $0,75^{* *}$ \\
\hline & $r_{E}$ & & & 0,17 & $-0,08$ & $-0,17$ \\
\hline Renfru & $r_{F}$ & & & & $-0,03$ & 0,03 \\
\hline & $r_{G}$ & & & & $-0,03$ & 0,04 \\
\hline & $r_{E}$ & & & & 0,12 & $-0,20$ \\
\hline Resfru & $r_{F}$ & & & & & $-0,14$ \\
\hline & $r_{G}$ & & & & & $-0,14$ \\
\hline & $r_{E}$ & & & & & $-0,09$ \\
\hline Alpta & & & & & & \\
\hline
\end{tabular}

*, ** Significativa $(P<0,05)$ y Altamente significativa $(P>0,01)$

Lonfru: Longitud de fruto; Nufrupta: Número de frutos por planta; Pefru: Peso de fruto; Renfru: Rendimiento de fruto; Resfru: Resistencia de fruto; Alpta: Altura de planta. 
parcial fenotípico (Tabla 4), se encontró correlación positiva y altamente significativa con el Pefru $(0,59 * *)$, más no con la Lonfru. Lo antes anotado, evidencia que el Nufrupta y Pefru tienen un efecto importante sobre el rendimiento de fruto, como lo sostienen Tavares et al. (1999), quienes señalan que la selección simultánea de ambos caracteres no debe ser descartada en selecciones indirectas, puesto que el mayor número de frutos conduce consecuentemente a frutos de menor peso.

Por otra parte, se identificaron correlaciones fenotípicas y genéticas de poco valor entre el Renfru y Resfru, lo que probablemente obedezca a un control genético independiente, dada su carencia de significancia y coeficientes de correlación cercanos a cero, como lo reportaron en maracuyá Espitia et al. (2008b).

El coeficiente de determinación $\left(\mathrm{R}^{2}\right)$ de los respectivos análisis de sendero (Tabla 5) fue de 0,60 y 0,62 y la magnitud del efecto residual (h) de 0,61 y 0,63, lo que indica que la variación de los efectos de la variable básica (Renfru), no es totalmente explicada por las variables explicativas utilizadas en el modelo. Resultados similares han sido reportados por Tavares (1999); De Carvalho et al. (1999, 2002) y Espitia et al. (2008a, 2008b) y esto se explica, posiblemente, porque otras variables no consideradas en el estudio están influyendo en Renfru, como el número de ramas productivas que es un carácter importante a tener en consideración para mejorar el rendimiento, ya que posee una alta coheredabilidad con el número de frutos y peso de frutos, igualmente moderada heredabilidad como tal, que permite avances genéticos, por la predominancia de la acción genética aditiva (Vadivel E Bapu 1988a, 1988b, 1990; Coimbra et al. 1999).

Las magnitudes y los sentidos de los efectos directos e indirectos derivados de la descomposición de las correlaciones fenotípicas y genotípicas para el Renfru en función de Lonfru, Nufrupta, Pefru y Alpta, están contenidas en la Tabla 5.

Tabla 4. Correlaciones parciales $\left(r_{\mathrm{FP}}\right)$ entre seis características agronómicas de berenjena, utilizando las correlaciones fenotípicas $\left(\mathrm{r}_{\mathrm{F}}\right)$.

\begin{tabular}{|c|c|c|}
\hline Pares de variables & $\mathrm{r}_{\mathrm{F}}$ & $\mathrm{r}_{\mathrm{FP}}$ \\
\hline Lonfru x Nufrupta & $-0,33$ & $-0,52^{*}$ \\
\hline Lonfru x Pefru & 0,39 & $-0,46^{*}$ \\
\hline Lonfru x Renfru & 0,00 & $0,38 \mathrm{~ns}$ \\
\hline Lonfru x Resfru & $-0,67^{* *}$ & $-0,77^{* *}$ \\
\hline Lonfru x Alpta & $0,52^{* *}$ & $0,56^{* *}$ \\
\hline Nufrupta x Pefru & $-0,63^{* *}$ & $-0,73^{* *}$ \\
\hline Nufrupta x Renfru & $0,56^{* *}$ & $0,80^{* *}$ \\
\hline Nufrupta x Resfru & $-0,02$ & $-0,45^{*}$ \\
\hline Nufrupta x Pefru & $-0,47^{*}$ & $0,26 \mathrm{~ns}$ \\
\hline Pefru x Renfru. & 0,04 & $0,59^{* *}$ \\
\hline Pefru x Resfru & $-0,06$ & $-0,36 \mathrm{~ns}$ \\
\hline Pefru x Alpta & $0,74^{* *}$ & $0,65^{* *}$ \\
\hline Renfru x Resfru & $-0,03$ & $0,32 \mathrm{~ns}$ \\
\hline Renfru x Alpta & 0,03 & $-0,19 \mathrm{~ns}$ \\
\hline Resfru x Alpta & $-0,14$ & $0,36 \mathrm{~ns}$ \\
\hline
\end{tabular}

*, ** Significativa $(P<0,05)$ y Altamente significativa $(P>0,01)$

Lonfru: Longitud de fruto; Nufrupta: Número de frutos por planta; Pefru: Peso de fruto; Renfru: Rendimiento de fruto; Resfru: Resistencia de fruto; Alpta: Altura de planta. 
Tabla 5. Análisis de los efectos directos (en negrita) e indirectos de la longitud de fruto (Lonfru), número de frutos por planta (Nufrupta), peso de fruto (Pefru) y altura de planta (Alpta) sobre el rendimiento de frutos (Renfru).

\begin{tabular}{|c|c|c|c|c|c|}
\hline \multirow{2}{*}{ VARIABLES } & \multicolumn{4}{|c|}{ CORRELACIONES FENOTÍPICAS } & \multirow{2}{*}{$\frac{r_{F}}{\text { con Renfru }}$} \\
\hline & Lonfru & Nufrupta & Pefru & Alpta & \\
\hline Lonfru & 0,17 & $-0,34$ & 0,21 & $-0,04$ & $-0,01$ \\
\hline Nufrupta & $-0,05$ & 1,02 & -0.44 & 0,04 & $0,56^{* *}$ \\
\hline Pefru & 0,05 & $-0,64$ & 0,70 & $-0,06$ & 0,04 \\
\hline \multirow[t]{4}{*}{ Alpta } & 0,09 & $-0,48$ & 0,52 & $-0,09$ & 0,03 \\
\hline & \multicolumn{4}{|c|}{$R^{2}=0,60 h=0,63$} & \\
\hline & \multicolumn{4}{|c|}{ CORRELACIONES GENOTÍPICAS } & $r_{G}$ \\
\hline & Lonfru & Nufrupta & Pefru & Alpta & con Renfru \\
\hline Lonfru & 0,19 & $-0,37$ & 0,24 & $-0,06$ & $-0,01$ \\
\hline Nufrupta & $-0,06$ & 1,05 & $-0,48$ & 0,05 & $0,56^{* *}$ \\
\hline Pefru & 0,06 & $-0,68$ & 0,75 & $-0,09$ & 0,04 \\
\hline \multirow[t]{2}{*}{ Alpta } & 0,10 & $-0,51$ & 0,57 & $-0,12$ & 0,04 \\
\hline & \multicolumn{4}{|c|}{$R^{2}=0,62 h=0,61$} & \\
\hline
\end{tabular}

** Significativos al $1 \%$ de probabilidad, respectivamente.

El análisis de sendero basado en el Renfru, como variable independiente, revela que los efectos directos (negrilla) del Nufrupta con una contribución de $(1,02$ y 1,05$)$ y de Pefru, con un aporte de $(0,70$ y 0,75$)$, explican el nivel de asociación con el Renfru. Estos resultados son coincidentes con los manifestados por De Carvalho et al. (1999), en ají y Lohakare et al. (2008), en berenjena, quienes cuantificaron efectos directos e indirectos por encima de 0,60, para estas dos variables y resaltan la necesidad de considerarlas en el mejoramiento simultáneo para rendimiento de frutos. El poco efecto del peso de frutos obedece, posiblemente, al hecho de cosechar esta hortaliza de manera temprana (inmaduro), antes de alcanzar su máximo peso, con el fin de atender y de preservar su demanda y calidad de mercado.

La Lonfru registró un efecto directo (negrilla) de 0,17 y 0,19 , respecto al Renfru, lo que destaca ausencia de relación causa efecto $y$, por lo tanto, sugiere que este carácter no es importante para mejorar el rendimiento de fruto, corroborando lo encontrado en la correlación genética y fenotípica (Tabla 2).
El efecto directo (negrilla) de Alpta sobre Renfru fue negativo $(-0,09$ y $-0,12)$, indicando que pocas ganancias genéticas pueden ser conseguidas en el proceso de selección por plantas de mayor porte, por su baja contribución al rendimiento de frutos, ello concuerda con lo encontrado por Légesse et al. (1999) y resaltan que los efectos indirectos, a través del Nufrupta y Pefru, influyen en rendimiento total de fruto, destacando la importancia de estas dos características en el mejoramiento del rendimiento de frutos en berenjena. De igual manera, el efecto residual es apreciable, lo que sugiere que el rendimiento de fruto no es totalmente explicado por los caracteres usados en el análisis de sendero y es muy probable que existan otras causas significativas influyendo sobre el rendimiento total de frutos, como lo indican Ingale $\&$ Patil (1995) y Arnhold et al (2006).

Se concluye, que las características número de frutos/ planta y peso/fruto son-las más importantes para el mejoramiento simultáneo por rendimiento de fruto. 
AGRADECIMIENTOS: A los productores de berenjena del caribe húmedo (Hortisinú), a Corpoica, a ASOHOFRUCOL, al Ministerio de Agricultura y Desarrollo Rural y a la Universidad de Córdoba, por la financiación para realizar esta investigación. Conflictos de intereses: El manuscrito fue preparado y revisado con la participación de todos los autores, quienes declaramos que no existe ningún conflicto de intereses, que ponga en riesgo la validez de los resultados presentados.

\section{BIBLIOGRAFÍA}

1. AGRONET, 2008. Área y producción agrícola y pecuaria. http://www.agronet.gov.co. Ministerio de Agricultura y Desarrollo Rural. (con acceso el 26/01/08).

2. ARAMENDIZ, H.; CARDONA, C.; JARMA, A.; ESPITIA, M. 2008. El cultivo de la berenjena (Solanum melongena L.). Universidad de Córdoba. Editorial Produmedios (Bogotá), 151p.

3. ARAMENDIZ, H.; HOYOS, F.; GARCÍA, E. 1999. Estimación de la variabilidad genética en una población criolla de berenjena (Solanum melongena L.) en el departamento de Córdoba. Temas Agrarios (Colombia). 4(8):117-125.

4. ARNHOLD, E.; MORA, F.; DEITOS, A. 2006. Correlaciones genéticas en familias $\mathrm{S}_{4}$ de maíz (Zea mays L.). Cien. Inv. Agr. (Brasil). 32(2):125-131.

5. BANSAL, S.; METHA, A.K. 2008. Genotypic correlation and path análisis in brinjal. National. J. Plant Improvement (India). 10(1):34-36.

6. BHATT, G.M. 1973. Significance of path coefficient análisis in determining the nature of the carácter association. Euphytica (Holanda). 22(2):338343.

7. COIMBRA, J.; GUIDOLIN, A.; DE CARVALHO, F.; COIMBRA, S.; MARCHIORO, V. 1999. Análise de trilha I: análise do rendimento de grãos e seus componentes. Ciência Rural (Brasil). 29(2):213-218.

8. CRUZ, C.D. 2004. Programa GENES. Versao Windows. Aplicativo Computacional em Genética e Estatística. Editora UFV. Universidade Federal de Viçosa. Disponible desde Internet en: http:// www.ufv.br/dbg/genes/genes.htm (con acceso el 16/01/05).

9. DE CARVALHO, C.; RODRIGUES, V.; CRUZ, C.; DIAS, V. 1999. Análise de trilha sob multicolinearidade em pimentao. Pesquisa Agropecuaria Brasileira. 34(4):603-613.

10. DE CARVALHO, C. ARRABAL, DE TOLEDO, J.; DE OLIVEIRA, M.; VELLO, N. 2002. Correlações e análise de trilha em linhagens de soja semeadas em diferentes épocas. Pesquisa Agropecuaria Brasileira. 37(3): 311-320.

11. DPN (Departamento Nacional de Planeación). 2005. La pobreza en el departamento de Córdoba. Características por grupo de municipios. Cartagena, junio de 2005. 17p. (Disponible en http://www.dnp. gov.co) (con acceso el 28/01/08).

12. ESPITIA, M.; ARAMENDIZ, H.; CADENA. J. 2008 . Correlaciones y análisis de sendero en algodón (Gossypium hirsutum L.) en el caribe colombiano. Rev. Fac. Nal. Agronomía (Colombia). 61(1):43254335.

13. ESPITIA, M.; ARAMENDIZ, H.; CARDONA, C. 2008b. Correlaciones para algunas propiedades físicas y químicas del fruto y jugo de maracuyá (Passiflora edulis var. flavicarpa Degener). Agronomía Colombiana. 26(2):292-299.

14. HABIB, H.; SADAQAT, S.; ASHFAQ, M.; AHMAD, R. 2007. Genetic association and path analysis for oil yield in sunflower (Helianthus annuus L.). Internal. J. Agr. E Biology (Pakistan). 9(2):359-361.

15. HATIPOĞLU, R. 1993. Introduction to biotechnology. Çukurova University. Book of Agriculture Faculty, Adana-Turkey. No. 129:4-5.

16. INGALE, B.V.; PATIL, S.J. 1995. Correlation and path analysis in brinjal. Indian J. Horticulturae. 52(1):55-59.

17. KRISHNA, A.N.; MUNSHI, A.; BEHERA, T.; SUREJA. A. 2007. Correlation and path analyses for yield and biochemical characters in snapmelon. Sabrao J. Breeding and Genetics (Filipinas). 39(1):65-72. 
18. KRUITEVA, L. 1985. Correlation in eggplant. Capsicum Newsletter (Italia) 4:82.

19. LOHAKARE, A.S.; DOD, V.M.; PESHETTIWAR, P.D. 2008. Correlation and path analysis studies in green fruited brinjal. Asian J. Horticulture (India). 3(1):173-175.

20. LEGESSE, G.; ZELLEKE, A.; BEJIGA. G. 1999. Character association and path analysis of yield and its components in hot pepper (Capsicum annum L.). Acta Agronómica Hungarica. 47(4):391396.

21. MAK, C.; VIJIARUNGAM, A.F. 1980. Variability in bacterial wilt resistance and interelationships of some characterics on brinjal (Solanum melongena L.). Sabrao J. Breeding and Genetics. 12(1):65-75.

22. MARTÍNEZ, O.; TORREGROZA, M. 1988. Análisis de sendero de componentes de rendimiento en ciclos de selección masal divergente por prolificidad en maíz. Revista ICA (Colombia) 23(3):200-208.

23. PALENCIA, G.; MERCADO, T.; COMBATT, E. 2006. Estudio agroclimático del departamento de Córdoba. Ed. Gráficas el Caribe, Montería. 126p.

24. PALOMO, I.; GUTIÉRREZ, M.; ASTUDILLO, L.; RIVERA, C.; TORRES, C.; GUZMÁN, L.; MOORECARRASCO, R.; CARRASCO, G.; ALARCÓN, M. 2009. Efecto antioxidante de frutas y hortalizas en la zona central de Chile. Rev. Chilena de Nutrición. 36(2):152-158.

25. RAIGON, D.M.; PROHENS, J.; MUÑOZ-FALCON, J.; NUEZ, F. 2008. Comparison of eggplant landraces and comercial varieties for fruit content of phenolics, minerals, dry matter and protein. J. Food Composition and Analysis. 21(5):370376.
26. SEKARA, A.; CEBULA, S.; KUNICKI, E. 2007. Cultivated eggplants-origin, breeding objectives and genetics resources a review. Folia Horticulturae (Polonia). 19(1):97-114.

27. SHIVANNA, J.; RAVIAND, C.S.; SREERAMU, S. 2007. Character associated and path coefficient analysis among economic traits in Makoi (Solanum nigrum L.). Karnataka J. Agricultura Science (India). 20(3):575-577.

28. SILVA, G.O.; VIEIRA, J. 2008. Componentes genéticos e fenotípicos para caracteres de importância agronômica em populaçao de cenoura sob seleçao recorrente. Horticultura Brasileira. 26(4):481-485.

29. TAVARES, M.; DE MELO, A.; SCIVITTARO, W. 1999. Efeitos diretos e indiretos e correlaçoes para caracteres relacionados com a produçao de pimentao. Bragantia (Brasil). 58(1):41-47.

30. VADIVEL, E.; BAPU, J.R. 1988a. Correlation studies in Solanum melongena L. Capsicum Newsletter. 7:84-85.

31. VADIVEL, E.; BAPU, J.R. 1988b. Heritability estimates in segregating generations of eggplant. Capsicum Newsletter. 7:86-87.

32. VADIVEL, E.; BAPU, J.R. 1990. Studies on coheritability for yield components in eggplant. Capsicum Newsletter. 8-9:66-67.

33. YADAD, D.S.; PRASAD, A.; SINGH, N.D. 1997. Characters association in brinjal (Solanum melongena L.). Indian J. Horticulturae. 54(2):171-175.

Recibido: Septiembre 12 de 2009

Aceptado: Febrero 18 de 2010 